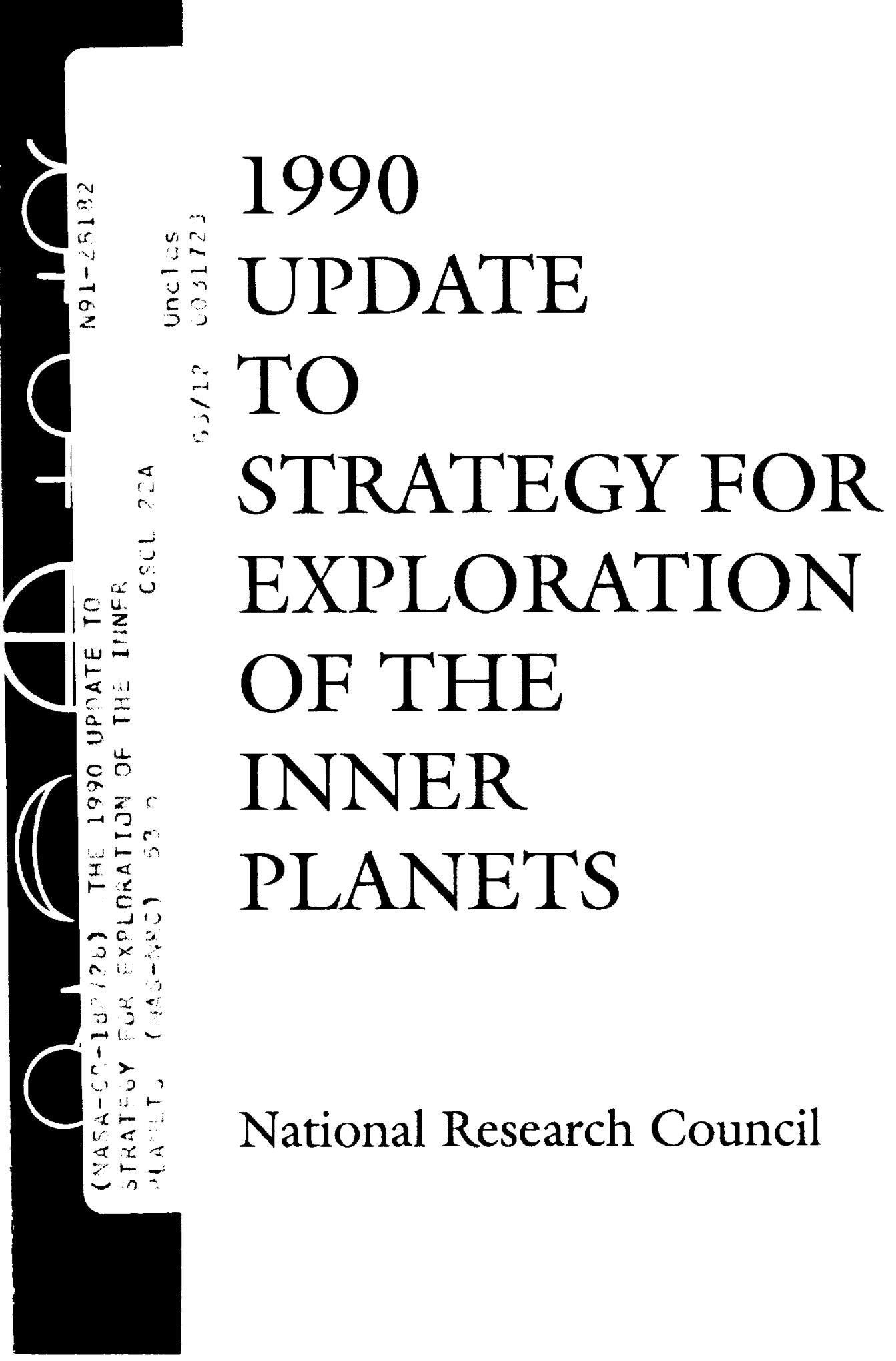





\section{0 \\ UPDATE \\ TO \\ STRATEGY FOR \\ EXPLORATION \\ OF THE \\ INNER \\ PLANETS}

\section{Committee on Planetary and Lunar Exploration \\ Space Studies Board}

Commission on Physical Sciences, Mathematics, and Applications

National Research Council

National Academy Press

Washington, D.C., 1990 
NOTICE: The project that is the subject of this report was approved by the Governing Board of the National Research Council, whose members are drawn from the councils of the National Academy of Sciences, the National Academy of Engineering, and the Institute of Medicine. The members of the committee responsible for the report were chosen for their special competences and with regard for appropriate balance.

This report has been reviewed by a group other than the authors according to procedures approved by a Report Review Committee consisting of members of the National Academy of Sciences, the National Academy of Engineering, and the Institute of Medicine.

The National Academy of Sciences is a private, nonprofit, self-perpetuating society of distinguished scholars engaged in scientific and engineering research, dedicated to the furtherance of science and technology and to their use for the general welfare. Upon the authority of the charter granted to it by the Congreas in 1863, the Academy has a mandate that requires it to advise the federal government on scientific and technical matters. Dr. Frank Press is president of the National Academy of Sciences.

The National Academy of Engineering was established in 1964, under the chaner of the National Academy of Sciences, as a parallel organization of outstanding engineers. It is autonomous in its administration and in the selection of its members, sharing with the National Academy of Sciences the responsibility for advising the federal government. The National Academy of Engineering also sponsors engineering programs aimed at meeting national needs, encourages education and research, and recognizes the superior achievements of engineers. Dr. Robert $M$. White is president of the National Academy of Engineering.

The Institute of Medicine was established in 1970 by the National Academy of Sciences to secure the services of eminent members of appropriate professions in the examination of policy matters pertaining to the health of the public. The Institute acts under the responsibility given to the National Academy of Sciences by its congressional charter to be an adviser to the federal govemment and, upon its own initiative, to identify issues of medical care, research, and education. Dr. Samuel 0 . Thier is president of the Institute of Medicine.

The National Research Council was organized by the National Academy of Sciences in 1916 to associate the broad community of science and tochnology with the Academy's purposes of furthering knowledge and advising the federal government. Functioning in accordance with general policies determined by the Academy, the Council has become the principal operating agency of both the National Academy of Sciences and the National Academy of Engineering in providing services to the government, the public, and the scientific and engineering communities. The Council is administered jointly by both Academies and the Institute of Medicine. Dr. Frank Press and Dr. Robern M. White are chaiman and vice chaiman, respectively, of the National Research Council.

Support for this project was provided by Contract NASW 4102 between the National Academy of Sciences and the National Aeronautics and Space Administration.

Additional copies of this repont are available from

Space Studies Board

National Research Council

2101 Constitution Avenue, N.W.

Washington, D.C. 20418

Printed in the United States of America 


\section{COMMITTEE ON PLANETARY AND LUNAR EXPLORATION}

Larry W. Esposito, University of Colorado, Chairman

Robert O. Pepin," University of Minnesota, Chaiman (1985-1988)

Andrew F. Cheng, Johns Hopkins University

Bruce M. Jakosky," University of Colorado

Jonathan I. Lunine, University of Arizona

Lucy-Ann McFadden, University of California, San Diego

Christopher P. McKay, NASA Ames Research Center

William B. McKinnon," Washington University

Duane O. Muhleman, California Institute of Technology

Philip Nicholson, ${ }^{*}$ Cornell University

Norman R. Pace, Indiana University

Gerald Schubert, University of California, Los Angeles

Peter H. Schultz, Brown University

Paul D. Spudis, USGS, Flagstaff

Peter H. Stone, Massachusetts Institute of Technology

G. Jeffrey Taylor, University of New Mexico

Richard W. Zurek, Jet Propulsion Laboratory

Staff

Paul F. Uhlir, Senior Program Officer

Mary Ellen Mack, Senior Secretary

LaVaughn Queen, Senior Secretary

\footnotetext{
"Former COMPLEX members who participated in writing this report.
} 


\section{SPACE STUDIES BOARD}

Louis J. Lanzerotti, AT\&T Bell Laboratories, Chairman

Philip Abelson, American Association for the Advancement of Science Joseph A. Burns, Cornell University

John R. Carruthers, INTEL

Andrea K. Dupree, Center for Astrophysics

John A. Dutton, Pennsylvania State University

Larry W. Esposito, University of Colorado

James P. Ferris, Rensselaer Polytechnic Institute

Herbert Friedman, Naval Research Laboratory (retired)

Richard L. Garwin, IBM T.J. Watson Research Center

Riccardo Giacconi, Space Telescope Science Institute

Noel W. Hinners, Martin Marietta Corporation

James R. Houck, Cornell University

David A. Landgrebe, Purdue University

John W. Leibacher, National Solar Observatory

Elliott C. Levinthal, Stanford University

Michael Mendillo, Boston University

William J. Merrell, Jr., Texas A\&M University

Richard K. Moore, University of Kansas

Robert H. Moser, The NutraSweet Company

Norman F. Ness, University of Delaware

Marcia Neugebauer, Jet Propulsion Laboratory

Joseph M. Reynolds, Louisiana State University

Sally K. Ride, California Space Institute

Robert F. Sekerka, Carnegie-Mellon University

Mark Settle, ARCO Oil and Gas Company

L. Dennis Smith, University of California, Irvine

Byron D. Tapley, University of Texas, Austin

Ex Officio

Albertus D. Welliver, The Boeing Company (Chairman, NAS/ASEB)

Staff

Dean P. Kastel, Staff Director

Richard C. Hart, Acting Associate Staff Director 


\section{COMMISSION ON PHYSICAL SCIENCES, MATHEMATICS, AND APPLICATIONS*}

Norman Hackerman, Robert A. Welch Foundation, Chairman George F. Carrier, Harvard University

Herbert D. Doan, The Dow Chemical Company (retired)

Dean E. Eastman, IBM T.J. Watson Research Center

Marye Anne Fox, University of Texas

Gerhart Friedlander, Brookhaven National Laboratory

Phillip A. Griffiths, Duke University

Neal F. Lane, Rice University

Christopher F. McKee, University of California at Berkeley

Richard S. Nicholson, American Association for the Advancement of Science

Jeremiah P. Ostriker, Princeton University Observatory

Roy F. Schwitters, Superconducting Super Collider Laboratory

Larry L. Smarr, University of Illinois at Urbana-Champaign

Norman Metzger, Executive Director

\footnotetext{
"The project that is the subject of this report was initiated under the predecessor group of the Commission on Physical Sciences, Mathematics, and Applications, which was the Commission on Physical Sciences, Mathematics, and Resources, whose members are listed in an appendix.
} 
2.: 


\section{Contents}

$\begin{array}{llr}1 & \text { Introduction } & 1 \\ 2 & \text { Overview } & 3 \\ 3 & \text { Mercury } & 7 \\ 4 & \text { Venus } & 11 \\ 5 & \text { The Moon } & 16 \\ 6 & \text { Mars } & 20 \\ 7 & \text { Supporting Research and Analysis } \\ 8 & \text { Program Balance } & 25 \\ 9 & \text { International Cooperation } & 28 \\ \text { APPENDIXES } & 30 \\ \text { A } & \text { Complete List of Recommendations Made } \\ & \text { List of Members of the Former Commission on } \\ \text { B } & \text { Physical Sciences, Mathematics, and Resources } & 35 \\ & & 47 \\ & & \\ & & \end{array}$





\section{1 \\ Introduction}

The responsibility of writing a strategy for exploration includes a corresponding responsibility to assess the extent to which its recommendations are carried out. It also requires a periodic review of the strategy to determine its relevance as its objectives are fulfilled and as the scientific field advances. In view of this advisory responsibility, the Committee on Planetary and Lunar Exploration (COMPLEX) has undertaken to review and revise the 1978 report Strategy for Exploration of the Inner Planets, 1977-1987 (National Academy of Sciences, Washington, D.C., 1978). The committee has found the 1978 report to be generally still pertinent, with some exceptions. COMPLEX therefore issues its new report in the form of an update. The previous report is not superseded, but modified as described below.

The committee reaffirms the basic objectives for exploration of the planets: to determine the present state of the planets and their satellites, to understand the processes active now and at the origin of the solar system, and to understand planetary evolution, including the appearance of life and its relation to the chemical history of the solar system.

This update incorporates as appropriate the advice provided by other relevant reports of COMPLEX and the Space Studies Board (SSB) during the period since the publication of the 1978 report. The committee completed the update pursuant to the following charge:

1. Identify those portions of the 1978 strategy that remain valid and those that require reassessment. 
2. Briefly summarize significant changes in the state of scientific knowledge regarding Mercury, Venus, the Moon, and Mars since the publication of the 1978 strategy.

3. Revise the scientific objectives and measurement requirements for those bodies as necessary.

4. Review the principal issues addressed by the 1978 strategy for the inner planets, including program balance, supporting research, and international cooperation.

Chapter 2 contains an overview of the committee's conclusions, including a listing of all recommendations. For each planet, a concise chapter outlines progress in the science since 1978, states the major scientific objectives, and provides a summary of recommendations; other relevant topics pertaining to each object are discussed as necessary (Chapters 3 to 6). In Chapters 7 to 9 the committee briefly addresses the broader policy issues mentioned in the charge. A complete listing of the 1978 recommendations appears as an appendix to this update. 
SIGNIFICANT CHANGES SINCE 1978

A number of developments in the decade following the publication of the 1978 report have made this update necessary. These developments fall into three areas-programmatic, scientific, and technical. The major programmatic issue is that progress toward the goals recommended in the 1978 report has been much slower than the committee anticipated when it wrote the original strategy. The Challenger disaster and cost increases associated with the Space Transportation System have resulted in long delays in the launch of planned missions. These unforeseen complications have placed large stresses on resources for new and planned missions, as well as on supporting activities. Since the publication of the previous strategy, only one U.S. mission has been launched to the inner planets. During this period the capabilities of other nations for planetary research have increased as well. This has provided a new environment for consideration of international cooperation.

The last decade has nonetheless included significant U.S. and USSR planetary missions. Pioneer Venus has orbited Venus, and the Soviet Union has launched six missions to Venus, including radar mappers, landers, and atmospheric balloons, and has sent the PHOBOS mission to Mars. Most recently, the United States has launched the Magellan mission, which is currently en route to Venus, and the Mars Observer mission is progressing toward a 1992 launch date. Galileo made new observations of Venus when the spacecraft flew by it on the way to Jupiter. Several European nations 
have contributed instruments to the Soviet VEGA and PHOBOS missions, and Japan has launched its first experimental mission to the Moon.

The major scientific developments include not only the understanding of new data from the space missions mentioned above, but also the deeper analysis of information from previous missions. The span of time has allowed a synthesis of many of these results. Additionally, basic research, ground-based observations, and theoretical modeling have provided new information and insights.

Technical advances in instrumentation and computation make possible investigations that were considered unrealistic a decade ago. One very positive development is that economical ways have now been found to send an orbiting spacecraft to Mercury, a project considered prohibitively difficult when the 1978 report was written.

The extensive delays in our planetary exploration program, the changed international environment, and the new data and continuing analysis of old data, together with our new perspectives based on current technology and understanding, all argue for a review of the existing strategy.

\section{REVIEW OF 1978 REPORT}

COMPLEX has undertaken a comprehensive review of the discussions and recommendations made in the 1978 report Strategy for Exploration of the Inner Planets: 1977-1987 (Appendix A). This review has included numerous presentations by leading authorities, who have described the current status and objectives of exploration of the inner planets. Based on these presentations and the most recent scientific publications, COMPLEX has concluded that the existing strategy is mostly valid and does not require extensive revision. The committee therefore endorses the discussion and recommendations of the 1978 report, except as noted below.

Nevertheless, some parts of the report are outdated, especially the descriptions of the state of scientific knowledge at the time of writing. The present report is therefore an update to the 1978 strategy, enumerating the significant progress since that time, providing a current perspective on the outstanding scientific objectives, and delineating a small number of recommendations that extend and modify the 1978 recommendations and measurement requirements. The major modifications are to raise the priority of some of the 1978 objectives, to note where expected achievement of some goals has not occurred, and to add some objectives not mentioned in 1978. The committee additionally provides advice on some key policy issues where significant developments have taken place over the last decade, in particular with regard to supporting research, program balance, and international cooperation. 


\section{NEW RECOMMENDATIONS}

The extensions and revisions to the recommendations made in the 1978 report are summarized in the following recommendations:

\section{Mercury}

- Characterization of Mercury's magnetic field should be a primary objective for exploration of that planet.

\section{Venus}

- Characterization of the basic structure, composition, and dynamics of Venus's atmosphere should be a primary objective.

- Acquisition of seismic data from Venus should be maintained as a highly desirable goal.

- Serious study of instruments operating at Venusian surface temperatures should be undertaken and preliminary studies should be conducted to determine the technical feasibility of sample return from Venus.

\section{The Moon} orbit.

- A spacecraft or series of them should be placed in a lunar polar

- The National Aeronautics and Space Administration (NASA) should develop the technology to deploy geophysical stations.

- Global mineralogical measurements at high spatial and spectral resolution should be given a high priority.

\section{Mars}

- The importance of the scientific objectives of study of the Martian atmosphere, interior, magnetic field, and global properties should be given equal priority with the objective of intensive study of local areas.

- The geochemical, isotopic, and paleontological study of Martian surface material for evidence of previous living material should be a prime objective of future in situ and sample return missions.

\section{Supporting Research}

- NASA should support a vigorous program of data analysis, basic research, and scientific instrument development. 


\section{Program Balance}

- Exploration of the inner planets in the next two decades should include further exploration of Mercury and Venus because a program of planetary exploration that includes only Mars and the Moon is scientifically inadequate.

\section{International Cooperation}

- Selection of foreign scientists and experiments for U.S. missions should be based strongly on scientific merit, and the free flow of scientific data and results should be a necessary precondition for any cooperative arrangements.

- NASA should consider all appropriate foreign capabilities available for planning and carrying out its missions and should cultivate those that enhance the scientific return.

- NASA should fully involve the scientific community in planning for international cooperation and in assessment of proposed cooperative missions. 


\section{3 \\ Mercury}

\section{PROGRESS}

Since the writing of the 1978 report, new discoveries have been made concerning Mercury's composition, atmosphere, and geology, and new insights have been gained into the planet's origin and evolution. One significant change in our understanding has resulted from our discovery of a variable tenuous atmosphere of $\mathrm{Na}$ and $\mathrm{K}$ by Earth-based, high-resolution spectroscopy. A component of this atmosphere may be derived from the vaporization of infalling meteoritic material, as well as from Mercury's regolith. It is potentially significant that Mercury's surface rocks may contain these relatively volatile elements. A reinterpretation of Mariner 10 ultraviolet (UV) spectrometer measurements indicates amounts of atmospheric oxygen comparable to that of Na. Photoionization and injection of $\mathrm{Na}$ ions into the magnetosphere may mean that $\mathrm{Na}$ is an important magnetospheric component. The $\mathrm{Na}$ atmosphere also has a significant electrical conductivity, and so magnetospheric currents can close in the $\mathrm{Na}$ atmosphere, allowing it to influence magnetospheric dynamics as does Earth's ionosphere. This coupling to the $\mathrm{Na}$ atmosphere has fundamental implications for magnetospheric processes, such as magnetic substorms and flux transfer events.

Improved understanding of surface composition has come from thermal emission measurements in the 7.2- to $11.2-\mu \mathrm{m}$ region. The globally averaged data so far indicate an intermediate silicate composition. New 
reflectance spectra in the visible and near-infrared (IR) show no $\mathrm{Fe}^{2+} \mathrm{ab}$ sorption band at $0.9 \mu \mathrm{m}$. The implication is that whatever basalts may exist on Mercury are extremely iron-poor, or that shock processing by impact masks this and other spectral absorptions.

Earth-based radar altimeter measurements show large scarps and regional elevation differences, confirming and quantifying the significant past tectonic activity revealed by Mariner 10 . Radio maps of the planet's surface can now determine regolith temperatures and, with sufficient analysis, regolith properties, such as dielectric constant and density averaged over broad areas.

Progress toward resolution of the question of volcanism on Mercury has been made as well. The global extent, depositional settings, and age relations of Mercurian smooth plains collectively indicate they are predominantly of volcanic origin. The plains within and surrounding the Caloris basin are, in particular, younger than Caloris, consistent with a volcanic origin. The plains outside are depressed in elevation, consistent with downwarping and the hypothesis that these plains create a gravity anomaly similar to that associated with lunar mascons. Systematic differences in crater shape and morphology suggest that signatures of both impact velocity and gravity exist, thereby providing an important comparison with other planets.

From a more theoretical standpoint, it is now recognized that tidal heating may play an important role for Mercury. Tidal heating could occur in a large solid inner core, supplying power to the planet's magnetic dynamo and prolonging the core's thermal evolution. Mercury's relatively large core may itself be due to a catastrophic mantle-stripping impact.

\section{SCIENTIFIC OBJECTIVES}

The primary objectives in the 1978 report were to determine the chemical composition of the planet's surface on both a global and regional scale, to determine the structure and state of the interior, and to extend the coverage and improve the resolution of orbital imaging. These objectives should be retained, but in addition, determination of the structure and time variation of the magnetic field should be elevated from a secondary to a primary objective.

\section{Surface Chemistry}

Determination of the major chemical components of Mercury's surface and characterization of regional variation in chemistry remain primary objectives. The composition of various surface and exposed deeper units remains unknown. This knowledge will also allow surface composition to 
be related to atmospheric composition. The bulk chemical analysis of the surface will provide a critical test of new cosmochemical and accretion theories by assessing the range of solar nebula conditions sampled by the innermost planet. Such an analysis will also show if the planet was chemically fractionated in a giant impact.

\section{Internal Structure and State}

Determining the extent and state of a dense central core on Mercury remains a primary objective. The extent of solid and/or liquid regions within the core is of particular interest to a more complete understanding of Mercury's interior chemistry, thermal evolution, spin-down history, and magnetic field generation. The composition of the core may vary from pure iron-nickel, and this too bears on Mercury's accretional history.

The 1978 report emphasized seismology. This remains a viable option, with penetrators providing a possible delivery system. The committee notes that the thermal environment on Mercury's surface is a severe challenge to the design of any instrumentation, and steps should be taken to address the technological issue. Deployment of seismometers would create an opportunity to also deploy other geophysical instruments, such as magnetometers and sensors for measuring the global heat flow, which is an important geophysical goal.

Consideration should also be given to the landing and tracking of passive radio beacons or other technologies that allow the obliquity and spin-axis libration to be determined. These values (along with knowledge of the second-degree gravity harmonics) could determine the size of a possible liquid core. This is somewhat analogous to the analysis of the Moon's librations using laser ranging data, which has yielded indirect evidence for a lunar liquid core.

\section{History of the Surface}

As recommended in the 1978 report, imaging should be extended to include the 50 percent of the planet not observed by Mariner 10 . Complete imaging, at a resolution of at least $0.5 \mathrm{~km}$, is necessary to understand Mercury's tectonic and possible volcanic evolution, to further characterize the impact cratering record in the inner solar system, and to broaden our understanding of the planet's overall geologic history. It would also provide an essential geologic context for interpreting surface chemistry measurements.

In the 1978 report, determinations of the global gravity and topography were stated as important, but secondary, scientific objectives. Such 
measurements would be of great use in understanding Mercury's geologic and geophysical history.

\section{Magnetic Field}

Characterization of the multipolar structure of the internal magnetic field has direct implications for the size and physical state of Mercury's core and for our understanding of the physical processes involved in the dynamo generation of the magnetic field. High-order multipole coefficients of the internal magnetic field and the temporal variability of these coefficients should be determined. The magnetospheric plasmas, energetic particles, and fields should be adequately characterized as well. This is important in its own right but also is necessary to provide the basis for separation of the internally generated magnetic field from the field generated by magnetopause and magnetospheric currents, or remanent surface fields.

\section{UPDATED RECOMMENDATIONS}

The updated strategy for exploration of Mercury differs from the strategy in the 1978 report in a number of important aspects. The 1978 report concluded that insertion of an appropriately instrumented planetological payload into a relatively low-altitude, circular orbit around Mercury required development of a low-thrust propulsion system. It has recently been demonstrated, however, that such a mission is feasible with conventional rocket launches and gravity assists at Venus and Mercury, thereby removing the perceived impediments to near-term orbital exploration of Mercury. Therefore, the 1978 report's recommendation that investigation of Mercury "be included ... later in the decade ... with the proviso that initiating such a mission does not inhibit or detrimentally affect the primary emphasis on the triad Earth-Mars-Venus" needs revision in two respects. A Mercury mission is a possible near-term activity, and justification of such a mission should rest on the important role of Mercury in understanding the origin and evolution of all the terrestrial planets.

The 1978 report relegated exploration of Mercury's magnetic field and magnetosphere to a secondary objective. Because of the direct connections between Mercury's magnetic field and the size and physical state of its core, determination of the multipole structure of the planet's magnetic field should be a primary scientific objective along with surface chemistry, internal structure, and imaging. Understanding how the Earth's geodynamo works is one of the major unsolved problems in geophysics; characterization of Mercury's magnetic field will provide crucial insights and constraints on dynamo theories. COMPLEX therefore recommends that characterization of Mercury's magnetic field be a primary objective for exploration of that planet. 


\section{4 \\ Venus}

\section{PROGRESS}

This section summarizes advances in our understanding of Venus since 1978 and outstanding problems that require resolution by future missions. The decade following the 1978 report witnessed the acquisition of the first geophysical data relevant to the planet's interior. The Pioneer Venus radar altimeter provided a global topographic map, and radio tracking of the orbiter supplied gravity data over limited regions. In contrast to Earth, Venus's gravity and topography are highly correlated, and interpretations of the combined data may involve mantle and lithosphere dynamical processes peculiar to Venus. The Pioneer Venus magnetometer placed a strict upper limit on any internally generated global magnetic field; the implications of this for the planet's internal structure and theories of the geodynamo are profound and include the possibility that the core is completely liquid.

The Soviet Venera 15 and 16 radar missions, as well as high-resolution Earth-based radar observations, have revealed a complex tectonic history for the Venus surface. There are unique features visible in these images that could have important implications for internal processes. Although the impact crater record provides a broad constraint on surface ages, limited resolution (1 to $2 \mathrm{~km}$ ) has prevented identification of the complete record with certainty. The Magellan mission should provide resolution sufficient to characterize the cratering history and to search for a possible relic crater population prior to climatic instability. The mission should also test hypotheses about the timing, style, and rate of surface-modification 
processes including erosion, possible fossil river beds, volcanic resurfacing, and perhaps plate tectonics.

Experiments to determine major element composition of surface rocks were performed by seven of the Soviet Venera landers. The elemental inventories are all consistent with two types of basalts: high-potassium basalt near a suspected impact feature and tholeiitic basalts in the lowland plains, similar to ocean floor basalts on Earth.

Noble gas abundances in the atmosphere of Venus were measured by the Pioneer Venus and Venera probes, but the data contain serious conflicts and are subject to profound differences of interpretation. The deuterium abundance in the atmosphere was tentatively determined from the 1978 Pioneer Venus data to be 0.016 . This elevated value has been a prime driver for the argument that the planet has lost a massive amount of water over the age of the solar system, although an alternative interpretation involving comet impacts is also viable. In addition, the reported abundance of water in the atmosphere has a very peculiar altitude profile when the Pioneer Venus and Venera measurements are combined. Since the water and the deuterium abundances are key quantities in understanding the evolution of the atmosphere and the planet's surface, improved abundances and height profiles are essential.

Global changes in the composition of the Venus atmosphere have recently been shown to occur on short time scales, on the order of months or years. Analysis indicates that the global average abundance of $\mathrm{SO}_{2}$ in the visible part of the atmosphere decreased tenfold between 1978 and 1983, perhaps as a result of the recovery of the atmosphere from a massive volcanic eruption.

Pioneer Venus provided important information on ionospheric and solar wind interactions, atmospheric thermal structure, zonal and meridional circulation, and planetary-scale waves and tides. The Soviet VEGA balloons collected additional information about the dynamics of the atmosphere at cloud level.

\section{SCIENTIFIC OBJECTIVES}

Measurements from Pioneer Venus, Venera, VEGA, and groundbased investigations have provided a quantitative data base but have also raised fundamental issues concerning the planet's interior, surface, and atmosphere that demand further study.

Determination of the interior structure is a primary objective for future exploration following successful completion of the Magellan mission. The existence or absence of a metallic core needs to be established, and the properties of the core-such as its radius, density, and physical state-must be determined. Knowledge of core mass and size will determine average 
mantle density and will constrain models of the composition of the interior. A seismometer array deployed by landers or penetrators on the surface would directly determine the planet's deep internal structure.

The necessity for a long-lived seismic array raises serious difficulties for instrument operation at the high temperatures of the Venusian surface. Development of reliable electronics to withstand operating temperatures of $500^{\circ} \mathrm{C}$ is required. Seismic probing would yield detailed radial profiles of seismic velocities and reveal any major density discontinuities in the core and mantle. It would be possible to determine if the planetary core were partially solidified as a test of explanations for the lack of a planetary magnetic field and theories of the geodynamo.

The development of high-temperature electronics for a long-term seismic array would also enable the establishment of a more general-purpose geophysical array to measure such quantities as surface heat flow-an important constraint on the bulk content of radiogenic elements in the interior. Long-term radio tracking of surface geophysical stations would reveal important characteristics of the planet's rotation, an additional source of information on internal structure and atmosphere-solid planet angular momentum exchanges. While Magellan may provide global topographic and gravity data, higher-resolution data on particular locations will be necessary to understand the interior dynamics and surface tectonic processes responsible for certain geological features. Should Magellan fail to collect the expected global topography and extensive gravity data, the acquisition of this information would remain a high-priority science objective.

Direct sampling of rock from a highland region has never been done and is crucial to determining whether such rocks have a granitic composition similar to that of continental shields on the Earth. Elemental analyses could be improved on future lander missions but require the development of electronics that can function at the high surface temperatures.

A complete elemental inventory along with mineralogical analysis would be key to interpreting the surface record and would provide important information on the chemistry of surface-atmosphere interactions on Venus. Such interactions are a critical component in the short- and long-term evolution of the atmosphere.

While important initial measurements of atmospheric dynamics and composition were made by the Venera spacecraft and by the Pioneer Venus orbiter and probe, some fundamental issues remain unresolved. New measurements of noble gas abundances, isotope ratios, molecular species, aerosols, and atmospheric dynamics are all needed.

In the upper atmosphere, the abundance of molecular oxygen is a key indicator of photochemical processes, but $\mathrm{O}_{2}$ has never been detected. The abundance of free $\mathrm{O}_{2}$ in the lower atmosphere would also be an important indicator of surface-atmosphere chemical equilibrium. Other 
photochemically important species that have not been measured or are poorly measured include compounds of $\mathrm{O}, \mathrm{Cl}$, and $\mathrm{S}$ above the cloud tops.

In the upper haze region, a number of questions remain after Pioneer Venus. Sulfur dioxide is a major absorber in the far-UV region of the spectrum, but additional work is needed to characterize absorbers in both the near- and far-UV ranges. Better measurements of these absorptions are vital for understanding the global heat balance and greenhouse effect. This requires high-resolution spectroscopy in the near-UV region, photometric and polarimetric studies of the bright and dark features, and laboratory work on cross sections.

Controversy also remains as to whether Pioneer Venus electric field data detected lightning. Whether lightning exists and whether it is generated in volcanic eruptions or in the cloud decks (which do not appear to precipitate or have large potential instabilities) are important open questions that should be addressed in future missions.

The dynamics of the superrotating Venus atmosphere needs additional study as well. What is the nature of the mean and temporally variable zonal and meridional circulations? What maintains the dominant superretrograde rotation? How do planetary-scale waves and tides transport heat and momentum? What is the interaction of cloud dynamics and circulation? Direct measurements of winds have utilized the motions of the Pioneer Venus and Venera descenders, but only at altitudes below $65 \mathrm{~km}$. Venusian winds can be measured to very high altitudes from future spacecraft using remote sensing techniques, including Doppler shifts in IR spectral lines and microwave rotational transitions of $C O$. LIDAR measurements should be developed for applications on both Venus and Mars. The aeronomy of the upper atmosphere, involving photochemistry and the wind fields, requires measurements well above the clouds. Temperature sounding and wind measurements are needed at all latitudes and heights for a comprehensive understanding of the circulation system. Finally, new measurements of ionospheric composition and dynamics are important to further advances in our understanding.

Approaches here include long-term UV imaging, remote temperature measurements in the cloud levels and above by a long-term orbiter, balloons and surface weather stations for circulation in and below the clouds, and theoretical efforts to develop three-dimensional global circulation and dynamical models.

\section{UPDATED RECOMMENDATIONS}

Essentially all of the strategy developed for Venus in the 1978 report remains valid. That report presumed that the Pioneer Venus and Venera investigations of the middle and lower atmosphere would completely 
determine its basic structure, dynamics, and chemical composition. The 1978 strategy therefore emphasized complementary investigations. The Pioneer Venus and Venera results, however, left some significant questions unanswered and raised important new issues. Hence, science objectives pertaining to middle and lower atmospheric composition and dynamics remain of primary importance, in addition to the objectives stated in the 1978 report. These were

1. to obtain a global map of the topography and morphology of its surface at sufficient resolution to allow identification of the gross processes that have shaped the surface;

2. to determine the major chemical and mineralogical composition of the surface material;

3. to determine the concentrations of photochemically active gases in the 65 - to $135-\mathrm{km}$ altitude region; and

4. to investigate the physical and chemical interactions of the surface with the atmosphere and the composition and formation of atmospheric aerosols.

Therefore, COMPLEX recommends that characterization of the basic structure, composition, and dynamics of Venus's atmosphere be a primary objective.

The 1978 report also considered study of the Venusian interior using passive or active seismic techniques as a secondary objective, while noting that it would be a primary objective if it appeared feasible. At that time, acquisition of such data required new technology development of instrumentation able to function for long durations at the surface temperatures. The current committee regrets to note that the required technology developments have not occurred in the intervening decade.

The committee wishes to elevate studies of the planet's interior to primary status, together with studies of the surface and the atmosphere, so that acquisition of seismic data is now regarded as a primary objective. The committee therefore reiterates the following recommendations of the 1978 report, restated as follows: that acquisition of seismic data from Venus be maintained as a highly desirable goal, that serious study of instruments operating at Venusian surface temperatures be undertaken, and that preliminary studies be conducted to determine the technical feasibility of sample retum from Venus.

Finally, the committee notes that a more sensitive search for any intrinsic magnetic field of Venus is of primary significance for determining the nature of the planet's interior. 


\section{5 \\ The Moon}

\section{PROGRESS}

The Moon is far more complicated than was generally appreciated when the 1978 report was written. This appreciation of the complexity of lunar history has come from continued study of lunar samples (including meteorites from the Moon), ground-based remote sensing data, sophisticated use of the Apollo orbital remote sensing data, and general advances in our understanding of geological and geophysical processes.

Besides being intrinsically interesting in its own right, the Moon provides a unique window into solar system history. Its origin is intertwined with that of Earth, its craters preserve a record of meteoroid fluxes through time, and it preserves at least a fragmentary record of its early (pre-fourbillion-year) evolution. It is one testing ground for our ideas about the origin and evolution of small planets. The Moon is an ideal body on which to study the processes, such as exogenic impacts, that have shaped the other solid bodies in the solar system and perhaps even caused extinctions of some forms of life on Earth. The Moon is also the only extraterrestrial body from which we have samples from a known geologic context, thereby providing a much more quantitative understanding of its history. The lunar soil preserves a four-billion-year-old record of the Sun's history. Finally, the Moon is a readily accessible body, making its scientific exploration easier to achieve.

Lunar science has evolved in several stages. The late 1960s and early 1970s focused largely on surface exploration, centering on the Apollo 
program. The mid-1970s through the 1980 s have emphasized reflection on those results in the context of new concepts and continued analysis. The future will address unsolved problems in lunar science and prepare for advanced studies from a lunar base.

During the mid-1970s, there was no consensus about how the Moon formed. Now the idea that it originated as a result of a giant impact on Earth has caught hold and has passed the tests given it so far. Nevertheless, this hypothesis is far from proven; assessing it requires a better understanding of the Moon's bulk chemical composition.

The idea that the primitive Moon was surrounded by an immense magma system known as the "magma ocean" continues to be a central tenet of lunar science, but vigorous debate is taking place about its nature and the processes that operated in it. Some investigators are even questioning whether there was a magma ocean. Proof of the magma ocean hypothesis hinges on the composition of the Moon's crust and the nature and ages of lunar anorthosites. Whether there was a magma ocean or not, it has become clear that there was a period prior to four billion years ago of intense igneous activity that modified the primordial lunar crust. In contrast to the narrow range of rock types defined during the mid-1970s, continued sample analysis has revealed a vast array of rock types in the lunar highlands, and remote sensing has shown that rock types rare in the Apollo collection are nevertheless abundant on the Moon. We need many more data from remote sensing and sample returns to determine the full range of rock types and how they relate to each other and to the products of the magma ocean.

Our understanding of mare basalts has advanced tremendously. After the Apollo missions ended, it was generally believed that mare basalt volcanism took place 3.2 to 3.8 billion years ago. Subsequent photogeological and lunar sample studies have shown that this type of volcanism occurred over a much greater time period, from 4.3 to possibly 1.0 billion years ago. This discovery has great implications for the Moon's thermal history. Furthermore, we now know that we sampled only about half of the full range of mare basalts. Because basalts contain information about the interior, we have an incomplete knowledge of the nature of the lunar mantle.

The time and rate of formation of large craters and the great lunar basins are still uncertain. During the mid-1970s, the consensus was that they formed during a narrow time interval 3.85 to 4.0 billion years agothe so-called lunar cataclysm. The consensus now is that the bombardment rate declined gradually and that only a few basins, which we happened to sample during Apollo, formed during the period from 3.85 to 4.0 billion years ago. The question will remain open, however, until samples are obtained and dated from basins far removed from those on the near side. In addition, the compositions and sources of the projectiles that made large 
craters and basins are largely unknown. Understanding these factors will contribute to our understanding of the later stages of planetary accretion with implications for the early history of the Earth.

Unanswered questions about the Moon abound. These problems can be addressed by global surveys from orbit, installation of a network of geophysical instruments, sample-return missions, and detailed field studies from a lunar base.

\section{SCIENTIFIC OBJECTIVES}

It is very important that the Moon's entire surface be adequately imaged and mapped geochemically, mineralogically, and geophysically. To meet this requirement, COMPLEX recommends that a spacecraft or series of them be placed in a lunar polar orbit. The measurements would benefit greatly if two spacecraft were in orbit simultaneously. The second spacecraft, which could be provided by another nation, would allow electromagnetic sounding of the interior and mapping of the far-side gravity field. Besides contributing to the solution of fundamental questions in lunar science, orbital measurements will provide critical information about where to locate a lunar base, regions containing potential resources, sites for sample-return missions and intensive field work, and emplacement of a network of geophysical stations.

To contribute to significant advances in lunar research, orbital measurements ought to include the following:

1. Abundances of major rock-forming elements $(\mathrm{O}, \mathrm{Si}, \mathrm{Fe}, \mathrm{Mg}, \mathrm{Al}$, $\mathrm{Ti}$, and $\mathrm{Ca})$ and of selected minor and trace elements $(\mathrm{K}, \mathrm{U}$, and $\mathrm{Th})$. This would yield the average composition of the surface and, if basins were used as natural drill holes, an estimate of the chemical composition of deeper crustal layers.

2. Spectroscopic measurements to obtain mineralogical and chemical data at high spectral and spatial $(<500-\mathrm{m})$ resolution. This would provide information on the distribution of lunar rock types.

3. Topographic data (combined with gravity data) to address problems involving density distributions and lithospheric loading.

4. Measurement of the gravity field on both the near and far sides to allow calculation of crustal thicknesses and densities.

5. Measurements of the Moon's magnetic field to shed light on the characteristics and origins of magnetic anomalies and to place constraints on the size of the core.

6. Imaging data to obtain global, digital photographic coverage with a line-pair resolution of $25 \mathrm{~m}$ and high resolution $(1 \mathrm{~m})$ of selected areas. These data would provide information about impact cratering, volcanism, 
and tectonism and would provide the geologic context needed to interpret other types of data.

7. Measurement of the average global surface heat flow to constrain the Moon's thermal history and provide indirect measurement of the bulk content of heat-producing elements.

A thorough understanding of the Moon will be impossible without knowledge of its interior. Better constraints on the size of the core will shed light on the origin of the remanent lunar magnetic field and, hence, on the origin of planetary dynamos in general. This will require the installation of a geophysical network of at least eight stations. Each station should include a seismometer, heat-flow probe, and atmospheric sensors. Such a seismic network would also be able to monitor the present meteoroid flux, using the entire Moon as a collecting surface. Deployment scenarios include automated landers or rovers, penetrators, or, eventually, astronauts. COMPLEX recommends that NASA develop the technology to deploy geophysical stations.

The lunar regolith contains a 4-billion-year-old record of solar particle emission history; deciphering this record will improve our understanding of how the Sun varies with time and improve the basis of predictions of how it will vary in the future. Lunar craters can be dated to provide a test of the hypothesis that mass extinctions of life on Earth were caused by periodic increases in the impact rate on our planet.

\section{UPDATED RECOMMENDATIONS}

The committee endorses the recommendations in the 1978 report. Measurement of the Moon's global chemical composition remains a high priority, but the committee recommends that global mineralogical measurements at high spatial and spectral resolution also be given a high priority. Much more information about the nature of the lunar interior is needed as well; acquisition of the appropriate geophysical data from orbit and on the lunar surface remains a high priority. This will require instrument development and research on how to deploy instruments on the surface. 


\section{6 \\ Mars}

\section{PROGRESS}

Although only one spacecraft, the short-lived Soviet PHOBOS mission, has encountered Mars since 1978, the last decade has produced a significant evolution of thought in many areas. Further, both the United States and the Soviet Union have begun to build new spacecraft.

Viking observations continued through 1982, and major advances in data analysis and modeling have added to our previous understanding of the planet. The discovery that SNC-class meteorites are likely pieces of Mars that have been transported to Earth possbily provides a direct sample of some part of the Martian surface. Viking orbiter imaging data provided as much as an order-of-magnitude increase in visible detail and facilitated a major increase in our understanding of the global stratigraphy and the geologic history of the planet. A broad picture of the evolution of the planet's surface since about 4 billion years ago has emerged, but there are still major uncertainties concerning the events in the earliest history of Mars, and about the nature and timing of specific geologic processes since then. Some of these include the mechanisms responsible for the global crustal dichotomy, the tectonic history within and outside of the Tharsis region, the chronicling of erosional processes, and the role and history of water in forming channel systems on the older and on younger terrains.

A global understanding of aspects of the climate system has also developed. Detailed analyses and modeling have been done of the seasonal variations of water vapor in the atmosphere and of the seasonal exchange 
and transport processes, the polar-cap energy balance, and an entire Mars year of atmospheric dust opacity measurements. Combined with a dramatic improvement in our understanding of and ability to model the atmospheric dynamics, these have led to major advances in our understanding of the atmospheric circulation and radiative processes. One important result was to establish that global dust storms occur during some years but not during others, with different dynamical regimes being represented. In conjunction with chemical analysis of the surface materials, these data also constrain the history of the near-surface deposits.

The distribution and properties of materials within the near-surface layer have also been mapped using thermal infrared and Earth-based radar remote sensing data and imaging observations of variable features. In combination with information on the abundance and transport of dust within the atmosphere, these data constrain the present Martian aeolian environment.

The search for life dominated the Viking lander science interests. The conclusions, after a fair amount of discussion, are that the data are most consistent with the absence of life. The complete absence of organic material in the soil is the strongest argument against the extant existence of biological material in the form with which we are familiar. Nevertheless, consistent with the SSB report Planetary Biology and Chemical Evolution: Progress and Future Directions (National Academy Press, Washington, D.C., 1990), the committee endorses the continued search for evidence of past life and biochemical evolution on Mars, as well as the continuing study of the history of water on Mars.

\section{SCIENTIFIC OBJECTIVES}

The 1978 strategy described five broad categories of prioritized primary scientific objectives for Mars. These were as follows:

1. The intensive study of local areas. Of special importance were the mineralogical, chemical, and petrological properties of the surface; the nature and chronology of major surface-forming processes; the nature of surface, subsurface, and atmospheric volatiles and their interaction with the surface; and the interactions of radiation with the surface.

2. The study of the structure and circulation of the Martian atmosphere. Global atmospheric and boundary layer processes were specifically called out, requiring orbital remote sensing and multiple ground stations measuring several meteorological parameters.

3. The study of the structure and dynamics of Mars's interior. Specific areas of study included topography and gravity field investigations, seismological study of the interior, and surface beacon tracking to directly determine the planet's precessional constant and thus its moment of inertia. 
4. The study of the nature of the Martian magnetic field and the character of the upper atmosphere, and their interactions with the solar wind. These processes and phenomena remain essentially unexplored.

5. The study of the global chemical and physical properties of the Martian surface. Measurements of these properties would provide constraints on the global crustal composition and its spatial variations, and would place the results of local intensive studies into a global context.

\section{UPDATED RECOMMENDATIONS}

Although the primary objective for Mars laid out in the 1978 report involved the intensive study of local areas, the intervening decade has seen a major research emphasis in areas addressed by some of the other objectives. This shift of emphasis was primarily fueled by analysis and modeling of Viking data, as well as additional Earth-based telescopic data. These studies showed the importance of global and regional processes on Mars, especially in comparison with similar processes on the Earth and Venus. Examples include the triggering of global dust storms, the history of the Martian climate, surface-atmosphere interactions affecting the history of volatiles, polar processes, and the role of the Tharsis uplift. From this perspective, while intensive local studies remain the single highestpriority objective, COMPLEX considers that the importance of the other four objectives has increased relative to their standing in the 1978 report and should now be treated as having equal priority.

Furthermore, the discussion of the first objective in the 1978 report and the quantitative specifications given there for the types and precision of measurements required to satisfy it point unambiguously to in situ investigations on the surface of the planet and to sample return. The objective can certainly be implemented in full only in this way. The committee notes, however, that a decade later the unexpected sensitivity and spatial and spectral resolution of several types of instruments are such that certain orbital measurements are capable of providing information on a local surface scale that bears on some aspects of this objective.

As described in the 1978 report, the detailed analysis of surface materials involved samples to be collected from a region within easy reach of a landed vehicle. The current scientific consensus is that such samples are no longer considered adequate to address this objective. Rather, samples need to be obtained from a variety of locations spread out over perhaps hundreds to thousands of kilometers, and they need to be identified with sufficient information to provide the geologic context for each sample. A collection of materials chosen from those present only in the immediate vicinity of a single landed vehicle would not be adequate.

The lowest-priority objective in the 1978 report involved the global 
chemical and physical characterization of the Martian surface. Since this task was nevertheless included as a primary scientific objective, the committee at that time explicitly recognized the importance of characterizing whole-planet, major regional-scale and intermediate-scale features, as distinguished from information at the local (a few kilometers) scale. However, only determination of whole-planet and major-unit chemical composition at low spatial resolution from orbit is called out specifically in the 1978 statement of measurement requirements. Remote sensing instruments that are either available now or are under development permit the committee to extend this objective to include regional and intermediate-scale surveys of surface mineralogy and physical properties (e.g., density and grain size)-a particularly important consideration for Mars, with its chemical and physical diversity at various scales. From its present perspective, the committee perceives this objective as being on an equal footing with objectives 2, 3, and 4 above.

Understanding of the spatial and temporal distribution of minor species in the Martian atmosphere would provide insight into the global atmospheric chemistry and the stability and evolution of the atmosphere. Photochemically produced species such as $\mathrm{CO}, \mathrm{O}_{2}$, and $\mathrm{OH}$ and species that evolve over time, such as those including deuterium, need to be measured at altitudes up to $80 \mathrm{~km}$. Direct measurements of the wind velocity profile as well as of surface winds are also required. An understanding of the global distribution of ice clouds is necessary due to its possible importance in the hydrologic cycle.

Because of the importance of the polar caps in controlling the present climate, their seasonal behavior needs to be better understood. Measurements of the energy balance and seasonally variable composition are required, along with observations pertinent to the interactions with the atmosphere, net annual loss or gain of different constituents from the caps, and year-to-year variations in their behavior. The importance of these processes in the evolution of the polar layered terrain and the implications of the structure of the layered terrain for understanding the integrated effects of these processes provide impetus for detailed study of the layered terrain as well.

Global Viking imaging at $100-\mathrm{m}$ resolution and some imaging at $10-\mathrm{m}$ resolution have not adequately and uniquely determined all details of the geologic history of the surface. Global compositional mapping at kilometer-scale resolution, combined with imaging of selected areas at higher resolution, would give us significant new information in this area.

Determination of the global and local heat flux from the interior, along with the presence or absence of an intrinsic magnetic field, would provide important constraints on the bulk composition and thermal history of the interior. 
As noted at the outset, observations of the surface and atmosphere of Mars were made in 1989 by the Soviet PHOBOS orbiter. Measurements made by several instruments were relevant to a better understanding of atmospheric and surface properties and composition, primarily in the equatorial regions, and have provided data on the Martian magnetosphere and its interaction with the solar wind. Although these data have not been completely analyzed, it is unlikely, due to the short mission lifetime and limited geographic extent of observations, that any of the major goals outlined in the 1978 report were completely addressed.

The U.S. Mars Observer mission is a near-polar orbiting spacecraft scheduled for launch in 1992, with global mapping to begin in 1993. Its goal is to map the surface and atmosphere for an entire Martian year.

If Mars Observer is successful, the original COMPLEX objectives of establishing the nature of the magnetic field and characterizing the global distribution of chemical and physical characteristics of the surface will be partly accomplished. In addition, contributions will be made to the objectives of exploring atmospheric structure and circulation, establishing the distribution of volatiles, and constraining the planet's interior structure by means of topography and gravity data. Mars Observer will also provide a base of data to guide the selection of sampling sites for a Mars sample return mission and to identify resources to be used in potential future human exploration of the planet.

The principal components of the 1978 report's objectives that will remain largely unaddressed after a successful Mars Observer mission are the prime objective of in situ (or remotely sampled) elemental, mineralogical, and petrological studies of selected areas; seismological and precessional studies of the interior structure of the planet; and the dynamical and chemical properties of the upper atmosphere and its interactions with the solar wind.

Also open will be the question of past life on Mars. If life developed in the more clement ages on Mars, it may have left chemical and fossil evidence. COMPLEX therefore recommends that the geochemical, isotopic, and paleontological study of Martian surface material for evidence of previous living material be a prime objective of future in situ and sample return missions. 


\section{7 \\ Supporting Research and Analysis}

The decade preceding the 1978 report included a fast succession of major and pioneering missions, with brief pauses for postmission synthesis. The 1978 report recommended both scientific instrument development and a "vigorous and ongoing program of data analysis and synthesis . . . designed to foster interdisciplinary and comparative planetological research." These recommendations have been implemented. The last decade also has witnessed, however, significant advances related to NASA's objectives that were stimulated not only by new mission data, but also by new ideas and unexpected discoveries.

Several examples can be cited. First, the discovery of meteorites from the Moon and Mars on the antarctic ice sheet provided unexpected new clues about planetary materials and raised critical questions about impact physics. This discovery and subsequent analysis have led to a new perspective on the transfer of materials between planets.

Second, the recognition of a geochemical cosmic signature on the Earth closely correlating with a period of dramatic biologic and climatic change led to a broader awareness of impacts as a fundamental geologic process, a fact already documented on the other planets. Further analyses raised the possibility for a nonrandom and perhaps even periodic flux of bodies affecting the inner solar system, a problem that can be uniquely addressed from future lunar studies.

Third, the timely convergence of lunar studies, enabling computer technology, and recognition of planet-scale collisions as possible events early in solar system history led to serious consideration of the Moon 
as a product of a catastrophic collision on Earth. Such a perspective now provides a new context for understanding lunar and perhaps early terrestrial history.

Finally, the discovery of a tenuous $\mathrm{Na}$ and $\mathrm{K}$ atmosphere around both the Moon and Mercury may provide a new means to investigate interactions of the surface with its environment (microimpacts), in addition to degassing from the interior. Such advances serve to underscore the progress in knowledge made possible by the continuity of a vital program of basic research.

What began as a philosophy of post-mission data analysis naturally evolved into inter-mission thematic analysis. This parallel approach of directly related mission definition and analysis and basic research has formed the foundation for posing testable questions, refining mission objectives, and revealing new or even unexpected directions. An integral part of this philosophy includes continuity of complementary studies and facilities.

Earth-based spectroscopic observations of the planets have provided significant information about planetary surface materials, while radar imaging of Venus has revealed important clues about the nature of its surface, providing baseline information for planning future missions.

Remote sensing of the planets will continue to be an important aspect of planetary studies. The capabilities of ground-based telescopes are rapidly improving. New long-term observatories in near-Earth orbit, such as the Hubble Space Telescope and the Space Infrared Telescope Facility, will allow higher resolution and sensitivity for remote planetary observatories. These improved remote sensing capabilities will provide a context for the space missions to the planets, as well as direct comparisons between simultaneous remote and local observations. The scientific return of the spacecraft activities will be enhanced by these concurrent observations from the ground or Earth orbit.

Laboratory analyses help to constrain interpretations or broaden understanding of complex phenomena. Such analyses cross a wide range of relevant problems, including remote sensing, impact cratering, shock physics, and surface-atmosphere interactions. Similarly, theoretical studies permit exploring at broad scales complex phenomena such as global climate models, celestial dynamics, and impact processes.

Analog planetary studies in terrestrial settings permit testing aspects of both the experimental and theoretical approaches. Moreover, they provide essential information for broad-scale geologic processes such as suboceanic volcanism and rifting. Finally, interdisciplinary themes and timely synergistic studies permit thoughtful study of new discoveries without the time-constrained analysis of missions.

Continuity in basic research not only helps to achieve one of NASA's most fundamental goals, but also establishes a healthy scientific climate for 
education of the next generation of planetary scientists. It is important that NASA maintain the appropriate balance between planetary missions and the supporting research and analysis that provide the basis for understanding new results and for training future researchers. COMPLEX therefore recommends that NASA support a vigorous program of data analysis, basic research, and scientific instrument development. 


\section{8 \\ Program Balance}

The question of the relative scientific priority for exploration of the inner planets is discussed in depth in the 1978 report. It emphasizes comparative planetology and the value of planetary studies in understanding the Earth. The report selects limited goals of high importance and focuses on selected planets. The three planets with atmospheres receive the highest priority because, as stated, the comparative planetology of these bodies is a key to understanding the formation and evolution of the Earth, and its atmosphere, oceans, and planetary processes.

The committee endorses the high priority of comparative planetology and believes that significant benefits may be derived from deeper study of Venus and Mars. Advances in our knowledge of the Earth and its environment for life are tied to progress in our understanding of the terrestrial planets as a class. The major environmental changes on Venus and Mars challenge our understanding of the terrestrial environment. Because of their similarities and valuable intercomparisons, the Earth, Venus, and Mars should be a special concern of solar system exploration within an overall balanced program.

Nevertheless, for a full understanding of the planets we need increased knowledge about the Moon and Mercury. These two objects provide unique information about planetary evolution and processes. They record on their surfaces the bombardment history in the inner solar system and give direct information on the processes of impact cratering. They also contain a record of their initial differentiation and magmatic activity prior to about 3.8 billion years ago-information mostly missing on Venus, Earth, and 
Mars. The history of planetary accretion and differentation is a major unsolved problem for all the inner planets. The magnetic field of Mercury is the example most similar to that of our own planet, and consequently, for a better understanding of the Earth's internal geodynamics, the interior of Mercury might provide valuable comparisons. Similarly, the Moon's small core may have generated a magnetic field for about a billion years; determining the size of the lunar core will help scientists understand how this field was generated. The low priority of Mercury in the 1978 report was partly based on the technical statement that orbiting missions to that planet were prohibitively difficult. This technical barrier has disappeared with the discovery of mission scenarios that use successive gravity assists from Venus and Mercury itself to reach orbit.

Thus, evolving scientific and technical understanding along with new discoveries has led to a more important status for the terrestrial bodies without atmospheres. Each object offers fruitful comparisons.

The scientific strategy for the inner planets may focus on one planet or several, but not to the complete exclusion of any one. This requirement holds even if NASA's commitment to manned exploration requires extensive scientific precursor studies of Mars and the Moon. The committee realizes that the broad goals of planetary science require a balanced program of basic science and exploration, which includes studies of all planets, the Moon, and select asteroids and comets in our solar system. The committee therefore endorses the statement from the 1978 report that on a time scale of two decades the general level of exploration for all the planets of the inner solar system should be brought into balance. The committee further recommends that exploration of the inner planets in the next two decades include further exploration of Mercury and Venus because a program of planetary exploration that includes only Mars and the Moon is scientifically inadequate. 


\section{$\stackrel{9}{\text { International Cooperation }}$}

At the time that the 1978 report was written, planetary exploration was a bipolar enterprise, conducted vigorously by the United States and the Soviet Union. The European Space Agency (ESA) had just been reorganized, and the space programs of Japan, India, and China were in their formative stages. It should therefore come as no surprise that the section on international cooperation in the 1978 report focused exclusively on making our scientific interactions with the Soviets more fruitful.

The situation has changed dramatically since the publication of the previous report, however. Research in space science now includes many nations. Western Europe and Japan have independent launch capabilities that can accommodate modest missions to our closest planetary neighbors. Western European nations in particular, both collectively through ESA and in many cases individually, have become important cooperative partners in American and Soviet planetary missions. Despite a four-year lapse in the U.S.-Soviet bilateral space agreement due to unrelated political issues, cooperation between the two nations formally resumed in 1986 and has attained unprecedented levels of success. In short, the committee's recommendations in the 1978 report have been either largely implemented in the context of U.S.-Soviet cooperation or rendered obsolete by the advent of multipolar exploration capabilities.

International cooperation has therefore become a major element in the planetary sciences. This is a positive development for a number of reasons in addition to the scientific concerns. Cooperation in planetary exploration can improve international relations, appeal to decision makers 
and thus enhance the desirability of a proposed mission, provide potential cost savings to the nations involved, and decrease the length of time between missions. From a scientific perspective, such cooperation broadens the participation by the scientific community, enhances communication and develops valuable contacts, promotes the vitality of the domestic and international scientific community, and allows optimum use of limited launch opportunities. For individual scientists, these opportunities span the gaps between their own nation's missions and provide some measure of continuity in new data and hardware development for training graduate students.

With appropriate planning and safeguards some drawbacks of this cooperative mode can be avoided. Cooperation naturally implies some loss of control and that the credit for scientific successes must be more broadly shared. Considerable effort in coordination must be invested, and this has to be balanced against the expected return. Ample preparation must be made for changes in the international climate and the possibility of technical failures that reduce a partner's ability to deliver its planned contribution.

Experience shows that the use of appropriate mechanisms on the national and individual level can often safeguard this process. The committee endorses the use of various mechanisms at different levels of cooperation, including bilateral and multilateral government agreements and memoranda of understanding, informal interagency coordinating groups, multinational science working groups, nongovernmental institutional agreements, scientist-to-scientist working relationships, and creative efforts by individual scientists to find productive international arrangements.

International cooperation necessarily develops in progressive stages, beginning with small, low-risk endeavors and proceeding to larger, more ambitious activities. The committee recognizes the present advantages of cooperation and encourages further progress with our international partners. As foreign agency programs and our own international activities become more robust, we should seriously consider joint planning and advisory operations with other nations, subject to appropriate concerns for reciprocity and significant contributions from all sides. Because all nations share similar scientific objectives for planetary exploration, care must be taken to avoid unnecessary duplication of space missions. It is now an appropriate time to consider mechanisms for sharing information and optimizing world resources for planetary exploration.

In pursuing any cooperative projects or programs, however, it is crucial that NASA maintain scientific integrity. Because the inclusion of foreign scientists and experiments on U.S. missions can displace American investigators, COMPLEX recommends that their selection be based strongly on scientific merit and that the free flow of scientific data and results be a necessary 
precondition for any cooperative arrangements. This will allow improved scientific return to be an additional advantage accruing from international cooperation. The formal mechanisms that have evolved for participation of scientists on the U.S. Mars Observer and the USSR Phobos mission provide a good example.

In view of the existing and growing scientific and technological capabilities now enjoyed by a number of nations and agencies for conducting inner planet exploration, NASA should consider all appropriate foreign capabilities available for planning and carrying out its missions and should cultivate those that enhance the scientific return. Such an approach will become increasingly important as more nations acquire their own means for spacecraft exploration.

Finally, the committee urges NASA to fully involve the scientific community in planning for international cooperation and in assessment of proposed cooperative missions. 
Appendixes 



\section{Appendix A \\ Complete List of Recommendations Made in the 1978 Report*}

\section{SCIENTIFIC GOALS, SEQUENCE, AND STRATEGY}

\section{Levels of Investigation, Goals, and Mission Techniques}

In order to provide more specific guidance to achieve the general strategy goals of planetary exploration, primary objectives, which are the principal basis for defining a mission, and secondary objectives, which greatly enhance the value of a mission, are presented. All specific missions will consist of both kinds of objectives. (p. 7 )

We reaffirm our earlier suggestion that NASA assess the acquisition procedures so that this distinction in objectives is apparent in the choice and management of experiments and Principal Investigators. (pp. 7-8)

We therefore recommend that an assessment of mission operations, including spacecraft control and scientific instrument and data management and the design and management of software control systems, be studied by the Agency at the earliest possible time and the evaluation be presented to the Committee. (p. 8)

"Excerpted from Strategy for Exploration of the Inner Planets: 1977-1987 (National Academy of Sciences, Washington, D.C., 1978).

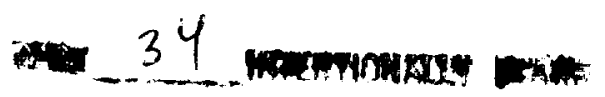




\section{Present Strategy}

As a consequence, the inner-solar-system strategy that we propose, like its counterpart for the outer solar system, is very conservatively paced and recommends only those goals that are of the highest importance and that can be achieved only by planetary encounter by spacecraft. (p. 9)

For the next decade there should be a shift in emphasis toward systematic exploration with emphasis on selected planets, but with some continuing level of reconnaissance to parts of the solar system where our ignorance is greatest and the opportunity for new discovery is large. (p. 9)

It remains the unanimous view of the Committee "that planetary exploration will continue to be an area of major scientific importance over the next decade and that continuing vigorous activity in this field is fully justified." (p. 9)

\section{Role of International Cooperation in Planetary Exploration}

We recommend that an effective mechanism be established by the United States and the Soviet Union for:

(a) The establishment of active open working relationships between Soviet and American scientists so as to discuss the scientific problems and to understand the nature and quality of the scientific experiments that might be carried out;

(b) The mutual identification of important scientific goals that are of a substantial nature;

(c) The early reciprocal communication between the United States and Soviet Union of the specific scientific objectives, both current and planned, in the area of planetary exploration;

(d) The establishment of concurrent commitments by both nations to achieve the goals in selected areas of mutual interest utilizing the agreed upon scientific objectives;

(e) The coordination of missions to a planet with full disclosure of mission planning and objectives so as to optimize the scientific contributions of both nations;

(f) The establishment of true cooperation between both nations through a reciprocal arrangement that allows the incorporation of significant scientific experiments by both parties on the same spacecraft; and

(g) The timely communication and exchange of information both during and after completion of a mission. (p. 12) 
We therefore recommend that progress in carrying out the above functions in planetary exploration be reviewed and assessed annually by segments of both governments. We request that the SSB be briefed on the results of the review so that progress in coordination and cooperation may be properly evaluated in the SSB yearly study of the Space Sciences Program. (p. 12)

We recommend that scientific planning, mission coordination, and cooperation between the United States and the Soviet Union be directed to those areas of planetary exploration where both nations have a vigorous and sustained effort and when the relative scientific strength and technological capabilities are mutually complementary in order to realize the maximum interest and scientific benefit. (p. 14)

\section{Launch Capabilities for Planetary Exploration}

We recommend that NASA adopt a policy that places the planning and development of launch capabilities for unmanned space exploration in a 10- to 20-year perspective and that focuses on the requirements of our long-term objectives. (p. 14)

We recommend that every effort be made to keep development of the Shuttle orbiter and a high-energy IUS on its nominal schedule. (p. 15)

"In the 1975 SSB report it was recommended that any intermediate upper stage (IUS) considered for transearth payloads have at least the same capability with regard to spacecraft payload and injection energy as presently exists and be sufficient to carry 500$\mathrm{kg}$ payloads with a C3 of $150 \mathrm{~km}^{2} / \mathrm{sec}^{2}$, payloads of $2000 \mathrm{~kg}$ at C3 of $90 \mathrm{~km}^{2} / \mathrm{sec}^{2}$, and payloads of more than $7000 \mathrm{~kg}$ for low C3." (p. 16)

In the 1975 report, the Committee endorsed the ongoing efforts by NASA to reduce the weight and cost of standardized available spacecraft without a corresponding reduction in capability using new developments in technology. (p. 17)

We recommend that a comparative assessment be carried out of solar electronic propulsion (SEP) design options in terms of feasibility, cost-effectiveness, and ability to meet the scientific objectives of solar-system exploration over the next two decades. The assessment should take into account currently stated objectives, and major emphasis should be given to the search for currently 
undiscovered exploration opportunities that may become available as a result of new propulsion capabilities. (pp. 18-19)

\section{Earth-Based and Earth Orbital Observations}

In the 1975 report, the Committee recommended that the adequacy of NASA support for the earth-based optical and radar programs, including aircraft and balloons for planetary observations, be reviewed regularly. The Committee was unable to conduct this review during its 1977 schedule but now recommends that this review be conducted no later than November 1978. (p. 20)

The order-of-magnitude improvement in spatial resolution available by use of the Space Telescope (ST), as well as access to wavelength regions unavailable from ground-based observatories, led us last year to "strongly recommend that a significant portion of the LST schedule be made available for planetary studies and that NASA begin immediate development of instruments that are oriented toward planetary studies for the LST and for other Shuttle deliverable payloads." COMPLEX wishes to reaffirm this view and to recommend further that the scheduling of ST observations be based on the potential scientific return from each proposed observational program with due regard for planetary mission planning and that the group charged with allocating telescope time be representative of the diverse interests of potential users. (p. 20)

\section{Planetary Quarantine Standards}

The Committee ... recommends that NASA conduct an overall review of its planetary quarantine policy and the policy for return and handling of samples from other planets to the earth in the light of our knowledge of these planets and in consonance with the terms of Article IX of the Outer Space Treaty. (p. 23)

\section{THE INNER PLANETS}

\section{Strategy for Study of Inner Solar System: 1977-1987}

As a statement of basic policy, the Space Science Board has defined the primary goals for investigation of the solar system. The primary goals are to determine the composition, structure, and environment of the planets and their satellites in order to define the present morphology and dynamics of the solar system 
and with the purpose of making major steps in understanding the processes by which the planets formed from the solar nebula and how they have evolved with time and how the appearance of life in the solar system is related to the chemical history of the system. The investigation of the interplanetary and interstellar medium is considered an intrinsic part of such an endeavor. (p. 31)

The Committee recommends that there should be a shift in emphasis toward systematic exploration of selected planets. The Committee also believes that some level of reconnaissance should be continued in accessible regions where our ignorance is greatest and where the opportunity of new discovery is large. (p. 31)

The Committee concludes that exploration of the inner solar system will be an undertaking of major scientific importance well beyond the next decade. (p. 32)

We have identified selected planets as the principal targets for investigation over the next decade in order adequately to fulfill the primary scientific goals enunciated by the Board and guarantee scientific advances in these areas rather than attempt to bring forward to a uniform level of exploration all the objects in the inner solar system. (p. 32)

On a time scale of two decades it is our view that the general level of exploration for all the planets of the inner solar system should be brought into balance. (p. 32)

The Committee has concluded that observation and measurement of the morphologic, physical, and chemical character of Mars, Venus, Mercury, and the moon on a global scale are of high general scientific importance and are basic to all planetological studies. (p. 33)

COMPLEX recommends that the triad of terrestrial planets, Earth, Mars, and Venus, should receive the major focus in exploration of the inner solar system for the next decade. The ultimate goal in this exploration is to understand the present state and evolution of terrestrial planets with atmospheres. The comparative planetology of these bodies is a key to the understanding of the formation of the earth, its atmosphere and oceans, and the physical and chemical conditions that lead to the origin and evolution of life. (p. 34)

The atmosphere-free terrestrial planets, Mercury and the moon, are complementary bodies of high scientific interest. (p. 34) 
The moon must remain an important object of exploration, which should receive strong consideration during the decade. (p. 35)

Steps should be made to prepare for the investigation of Mercury after definition of an adequate propulsion capability and in advance of availability of the system. (p. 35)

Subsequent to the development of an adequate transportation system, it is the view of COMPLEX that more substantial resources should be diverted toward the accomplishment of the major objectives of planetary exploration. (p. 35)

\section{Policy Considerations for Sample Retum}

In order to carry out an adequate program of exploration of the solar system, there will be a need to return several times to some planets with different spacecraft carrying different experiments, over a period of two decades. (p. 40)

\section{Conclusion, Summary, and Recommendations}

As a result of this examination, COMPLEX recommends the following:

1. Sample return from solar-system bodies should be considered a mission technique within the framework of a continuing program of scientific exploration and not a terminal, longterm goal.

2. Studies should be initiated to develop the special technology for such sample returns, with the moon, Mars, and Venus being examples of prime candidates for such sample return.

3. In situ investigations should continue to be a major mission mode for planetary exploration.

4. Efforts should be undertaken (as described in Chapter 6) to identify and develop the more advanced scientific instrumentation and manipulative techniques needed for the scientific objectives of future in situ planetary investigations. (p. 41)

\section{The Role of Global Maps}

A global map or image of the surface of a planet at good resolution is considered to be a major scientific contribution and is basic to any advance in the understanding of the terrestrial planets. (p. 42)

We do not wish to imply that global images at increasingly high resolutions are the means for continuing planetary exploration, 
but rather that an adequate resolution global image provides a fundamental framework for carrying out the specific analytic experiments on planetary bodies. The quantitative, analytic, and observational experiments must undoubtedly be the major means of scientific advance in an era of intensive exploration. (p. 43)

\section{Objectives for Mars}

We recommend that intensive study of Mars by spacecraft be achieved within the period 1977-1987. (p. 43)

\section{Primary Objectives}

In summary, the primary objectives in order of scientific priority for the continued exploration of Mars are (1) the intensive study of local areas (a) to establish the chemical, mineralogical, and petrological character of different components of the surface material, representative of the known diversity of the planet; (b) to establish the nature and chronology of the major surface forming processes; (c) to determine the distribution, abundance, and sources and sinks of volatile materials, including an assessment of the biological potential of the Martian environment, now and during past epochs; (d) to establish the interaction of the surface material with the atmosphere and its radiation environment; (2) to explore the structure and general circulation of the Martian atmosphere; (3) to explore the structure and dynamics of Mars's interior; (4) to establish the nature of the Martian magnetic field and the character of the upper atmosphere and its interaction with the solar wind; (5) to establish the global chemical and physical characteristics of the Martian surface.

These objectives are multiply connected. (p. 44)

\section{Strategy for Mars}

... the global and in situ studies of the planet and the return of Martian material are complementary components of an overall program of investigation; each of the components is separately necessary. (p. 49)

We note ... that although a specific sequence of investigations, namely global and in situ studies followed by sample return, is desirable, it is not necessary. (p. 49)

We ... recommend that detailed exploration, on both global and local scales, of the diverse environments of Mars for purposes of 
understanding surface, near-surface, and atmospheric processes is a worthy goal in its own right and should be accomplished within the next decade. (p. 49)

We ... reaffirm our view that the return of unsterilized surface and subsurface samples to Earth is a major technique for the exploration of Mars. (p. 50)

\section{Objectives for Venus}

The Committee ... recommends that there be continued exploration of Venus by spacecraft during the next decade. (p. 55)

\section{Primary Objectives}

The primary objectives of the exploration of Venus during the period 1977-1987, beyond the Pioneer Venus mission, in order of importance, are (1) to obtain a global map of the topography and morphology of its surface at sufficient resolution to allow identification of the gross processes that have shaped the surface, (2) to determine the major chemical and mineralogical composition of the surface material, (3) to determine the concentrations of photochemically active gases in the $65-135 \mathrm{~km}$ altitude region, and (4) to investigate the physical and chemical interactions of the surface with the atmosphere and the composition and formation of atmospheric aerosols. (p. 57)

We further recommend that some images be taken of a limited number of selected regions at a substantially higher resolution than indicated above. (p. 57)

The Committee has considered some possibilities and concludes that a serious study effort of the technology of instruments operating at Venusian surface temperatures and pressures must be undertaken before any particular approach is recommended. The substantial effort should be initiated in this area at the earliest possible time. (p. 58)

\section{Secondary Objectives}

COMPLEX believes that acquisition of seismic data from Venus should be maintained as a visible and highly desirable goal. (p. 58)

We recommend that preliminary studies be undertaken to determine the technical feasibility of returning samples of materials from the surface of Venus in order to ascertain the significance 
of this technique in developing an effective strategy for Venusian exploration over a time period of 20 years. (p. 59)

\section{Strategy and U.S.-USSR Cooperation}

It is the view of this Committee that continued scientific exploration of Venus offers an ideal arena for cooperation between the Soviet Union and the United States. We recommend that cooperative efforts be undertaken according to the precepts set forth earlier in this report. (p. 59)

Thus we recommend that a first U.S.-USSR collaboration proceed through the independent development of complementary experiments to be flown on a single spacecraft and plan for an early collaborative launch. (p. 60)

Studies of Venus will make a crucial contribution to our understanding of the behavior and evolution of terrestrial environments. As a result, studies of Venus must be pursued vigorously regardless of the fate of attempts to make it a cooperative endeavor. (p. 60)

\section{Objectives for Mercury}

\section{Scientific Objectives}

The primary planetary objectives in the exploration of Mercury for the period 1977-1987 are to determine the chemical composition of the planet's surface on both a global and regional scale, to determine the structure and state of the planet's interior, and to extend the coverage and improve the resolution of orbital imaging. (p. 68)

\section{Imaging}

Secondary planetary objectives of Mercury explorations are (1) further exploration of Mercury's magnetosphere and internal magnetic field, (2) measuring global heat flow, and (3) conducting gravity and topographic surveys of the planet. (p. 69)

\section{Conchusion}

We recommend that the planet then be included as an element in the sequence later in the decade and that the objectives as stated be adopted as the guideline for mission planning to implement the strategy, but with the proviso that initiating such a mission 
does not inhibit or detrimentally affect the primary emphasis on the triad Earth-Mars-Venus. (p. 71)

\section{Objectives for the Moon}

Scientific Objectives

The primary scientific objectives for exploration of the moon by spacecraft in the period 1977-1987, in order of importance, are (1) to determine the chemistry of the lunar surface on both a global and regional scale; (2) to determine the surface heat flow on both a global and a regional scale; and (3) to determine the nature of any central metallic core in the moon. (p. 72)

\section{U.S.-USSR Cooperation}

We recommend that formal negotiations to explore the possibility of such coordinated lunar missions be initiated. (p. 74)

\section{Fields and Particles for Inner Solar System}

We recommend that the determination of the strength and character of the internal magnetic fields (both global fields and, where possible, small-scale remanent fields) be an important goal in the exploration of planetary bodies. (p. 75)

We recommend that both cruise and orbiting phases of planetary missions be utilized for the conduct of important interplanetary and solar measurements when such an approach offers a significant increment over other available techniques and with due regard for a balanced approach to the first-order goals of such missions. (p. 76)

The Committee regards an understanding of the fundamental processes governing the solar wind's interaction with planets as a major goal of solar-system exploration and recommends that a global characterization be obtained of each planet's interaction with the solar wind. (p. 76)

\section{Minor Bodies Investigations}

\section{Meteorites}

COMPLEX strongly recommends continued intensive studies on meteorites as a parallel effort to the study of lunar samples, to earth-based observations of the solar system, and to space missions in the study of the planetary system. (p. 81) 


\section{Conclusions}

In order to develop properly the scientific objectives and the relative place that direct exploration of these objects should have in the overall strategy, as separate bodics and as a class of objects, COMPLEX recommends that a special study be carried out during the next year. (p. 81)

COMPLEX calls attention to its 1975 recommendation "that efforts be directed toward establishing the nature and quality of scientific experiments that could yield important data in a comet encounter so that the role of a comet investigation can be properly assessed in the framework of the current strategy." (p. 81)

\section{SCIENTIFIC INSTRUMENTATION DEVELOPMENT}

We recommend that a mechanism be established by NASA to bring this knowledge to bear on planetary problems more effectively. Instrumentation studies, organized by NASA, which focus on specific instrument requirements and on present and future problem areas, would be an effective first effort. (p. 86)

\section{POSTMISSION DATA ANALYSIS AND SUPPORTING RESEARCH}

We ... recommend that NASA establish a vigorous and ongoing program of data analysis and synthesis, which is designed to foster interdisciplinary and comparative planetological research. (p. 89)

We further recommend that NASA arrange for each mission science team to provide within a reasonable time frame a comprehensive report of its experiments and experimental results along with extended interpretations of these observations. (p. 89)

\section{EVALUATION OF PROPOSED PROGRAMS}

\section{Interdisciplinary Scientist Statement}

COMPLEX unanimously agrees that every proposed planetary mission should follow the lead of Atmosphere Explorer and Pioneer Venus in having interdisciplinary scientists appointed to the mission Science Steering Group. (p. 91) 


\section{STATUS OF EXISTING PROGRAMS}

\section{Viking-Extended Mission}

COMPLEX recommends that the meteorology and imaging experiments on both landers and the seismometer on Lander 2 be continuously monitored and that the ranging to the Viking landers be continued for as long as possible. (p. 93)

COMPLEX recommends that NASA maintain adequate ground support (personnel, tracking, computers, and image processing facilities) for the lifetime of the orbiters to ensure a maximum return of imaging data. (p. 93)

The Committee recommends that special emphasis be given to laboratory studies aimed at deciphering the data obtained by Lander biology experiments. (p. 94)

COMPLEX recommends that full advantage be taken of opportunities during the Viking Extended Mission to carry out a series of close encounters with the outer satellite, Deimos, in order to determine its mass and mean density. (p. 94)

\section{Pioneer 11}

The value of the Pioneer 11 scientific role in exploring the outer solar system led COMPLEX to recommend in 1975 that "the coverage of Pioneer 11 be restored to a sufficient level by mid-1979 in order to optimize the science return at the Saturn encounter." (p. 96)

\section{Role of Lunar Samples and Data in Planetary Science}

We view lunar sample research and lunar data analysis and synthesis as essential elements of planetary studies and therefore recommend that these elements continue to receive substantial support as an integral and unique element of the exploration of the inner solar system. (p. 97) 


\section{Appendix B \\ List of Members of the Former Commission on Physical Sciences, Mathematics, and Resources}

Norman Hackerman, Robert A. Welch Foundation, Chairman

Robert C. Beardsley, Woods Hole Oceanographic Institution B. Clark Burchfiel, Massachusetts Institute of Techology George F. Carrier, Harvard University Ralph J. Cicerone, University of California at Irvine Herbert D. Doan, The Dow Chemical Company (retired) Peter S. Eagleson, Massachusetts Institute of Technology Dean E. Eastman, IBM T. J. Watson Research Center Marye Anne Fox, University of Texas Gerhart Friedlander, Brookhaven National Laboratory Lawrence W. Funkhouser, Chevron Corporation (retired) Phillip A. Griffiths, Duke University

Neal F. Lane, Rice University Christopher F. McKee, University of California at Berkeley

Richard S. Nicholson, American Association for the Advancement of Science Jack E. Oliver, Cornell University Jeremiah P. Ostriker, Princeton University Observatory Philip A. Palmer, E. I. du Pont de Nemours \& Company Frank L. Parker, Vanderbilt University Denis J. Prager, MacArthur Foundation David M. Raup, University of Chicago Roy F. Schwitters, Superconducting Super Collider Laboratory Larry L. Smarr, University of Illinois at Urbana-Champaign Karl K. Turekian, Yale University 
$\longrightarrow$ 